\title{
Validation of a simple computed tomography scoring system to predict the malignant nature of pleural effusion
}

\author{
Rania A. Sweed, Ayman I. Baess
}

\begin{abstract}
Objective We aimed to validate a computed tomography (CT) scoring system and assess its sensitivity and specificity to predict the malignant nature of pleural effusion that is exudative and of undetermined origin.
\end{abstract}

\begin{abstract}
Patients and methods This is a retrospective study that enrolled 123 patients who were referred for medical thoracoscopy in the Chest Department, Alexandria Main University Hospital, between 2013 and 2017 for diagnosing exudative pleural effusion of undetermined origin. CT scans were reviewed by a radiologist who was blinded to the final diagnosis. We applied a scoring system that was generated by Porcel et al. Scoring results were then evaluated using the final diagnosis of thoracoscopic pleural biopsies as the reference.
\end{abstract}

Results The CT score showed a sensitivity and a specificity of 70 and $66.7 \%$, respectively, with an negative predictive value $83 \%$ and a positive predictive value $48 \%$, and the area under the receiver operating characteristic curve was 0.745 .using a cut-off value of at least 7 .

\section{Introduction}

Pleural effusion has been known to be a clinical challenge as differentiating benign from malignant effusion is not an easy task [1-3]. Contrast computed tomography (CT) is an important step in the diagnostic pathway of undiagnosed exudative pleural effusions [4]. Nevertheless, the value of CT in differentiating between benign and malignant causes of pleural disease is undetermined [5]. Few studies have explored the value of $\mathrm{CT}$ in predicting the malignant nature of pleural effusions [6-8]. This study aimed to validate a CT scoring system and assess its sensitivity and specificity to predict the malignant nature of pleural effusion that is exudative and of undetermined origin.

\section{Patients and methods}

This is a retrospective study that enrolled 134 patients who were referred for medical thoracoscopy in the Chest Department, Alexandria Main University Hospital, between 2013 and 2017 for diagnosing exudative pleural effusion after failure to reach a definite diagnosis using noninvasive workup. Patients of both sexes were included, aged 18 years or older, and presenting with exudative undiagnosed pleural effusion either unilateral or bilateral. Patients unfit for medical thoracoscopy, patients suffering from pleural effusion with extensive loculations, patients weighing more than $120 \mathrm{~kg}$, patients whose data were incomplete, and finally patients whose final
Conclusion The CT scoring system could not predict the malignant nature of exudative effusion with great accuracy. Egypt J Bronchol 2019 13:710-715 (C) 2020 Egyptian Journal of Bronchology

Egyptian Journal of Bronchology 2019 13:710-715

Keywords: computed tomography scoring system, malignant pleural effusion, predictors of malignancy

Department of Chest Diseases, Faculty of Medicine, Alexandria University, Alexandria, Egypt

Correspondence to Dr. Ayman Baess, MD, PhD, Department of Chest Diseases, Faculty of Medicine, Alexandria University, Alexandria, 21613, Egypt. Tel: +20 100682 2068; fax: +20 3485 3961; e-mails: ayman.baeis@yahoo.com, ayman.baeis@alexmed.edu.eg

Received: 22 May 2019 Accepted: 5 September 2019 Published: 21 January 2020

diagnosis was inconclusive were excluded from our study and the final number of patients who fulfilled our inclusion criteria was 123 patients.

Demographics, clinical data, laboratory investigations, and radiology of all patients were reviewed as well as the final diagnosis on the basis of histopathological and microbiological examination results of pleural biopsies obtained using medical thoracoscopy. The initial workup included plain radiograph of the chest postero-anterior (PA) view, pleural tapping, and analysis of pleural fluid (chemical, bacteriological smears and cultures, and cytopathological examination). CT scans were reviewed by a radiologist who was blinded to the final diagnosis. We applied a scoring system that was generated by Porcel et al. [8] (score sum ranging from 0 to 20; Table 1). Scoring results were then evaluated using the final diagnosis of thoracoscopic pleural biopsies as the reference. The performance parameters of the CT scoring system were evaluated including sensitivity, specificity; positive predictive value (PPV), negative predictive value (NPV), and area under receiver operating characteristic curve.

This is an open access journal, and articles are distributed under the terms of the Creative Commons Attribution-NonCommercial-ShareAlike 4.0 License, which allows others to remix, tweak, and build upon the work non-commercially, as long as appropriate credit is given and the new creations are licensed under the identical terms. 
Table 1 Computed tomography scan score for predicting the malignant etiology of pleural effusions

\begin{tabular}{lc}
\hline Parameters & Score \\
\hline Any pleural lesion $\geq 1 \mathrm{~cm}^{\mathrm{a}}$ & 5 \\
Liver metastases & 3 \\
Abdominal mass & 3 \\
Lung mass or lung nodule/s $\geq 1 \mathrm{~cm}$ & 3 \\
Absence of pleural loculations & 2 \\
No pericardial effusion & 2 \\
Nonenlarged cardiac silhouette & 2 \\
\hline
\end{tabular}

${ }^{a}$ Any pleural lesion (i.e. nodule, mass, or thickening).

\section{Statistical analysis of the data}

Data were fed to the computer and analyzed using the IBM SPSS software package version 20.0. (IBM Corp., Armonk, New York, USA). Qualitative data were described using number and percent. Quantitative data were described using range (minimum and maximum), mean, SD, and median. The significance of the results obtained was judged at the $5 \%$ level. The $\chi^{2}$-test was used for categorical variables, to compare between different groups. Fisher's exact or Monte Carlo correction was used for correction for $\chi^{2}$ when more than $20 \%$ of the cells have an expected count less than 5. The Student $t$-test for normally distributed quantitative variables was used to compare between two studied groups. The Mann-Whitney test was used for abnormally distributed quantitative variables to compare between two studied groups. A receiver operating characteristic curve (ROC) was generated by plotting sensitivity (TP) on the $y$-axis versus 1 -specificity (FP) on the $x$-axis at different cut-off values. The area under the ROC curve denotes the diagnostic performance of the test. Area more than $50 \%$ yields acceptable performance and area about $100 \%$ is the best performance for the test. The ROC curve also allows a comparison of performance between two tests. Results were judged at a level of significance of $5 \%$.

\section{Results}

We reviewed the data of 134 patients who underwent medical thoracoscopy during the period 2014 and 2018. We enrolled only 123 patients who fulfilled our inclusion criteria. The majority of the patients (70.7\%) were finally diagnosed with malignant pleural effusion (Fig. 1). Demographic and clinical data of the patients studied are shown in Table 2. Patients with malignant disease were older than those with benign disease. Patients with malignant pleural effusion experienced cough, chest pain, and weight loss more significantly compared with patients with pleural effusion of benign nature. However, patients with benign pleural effusions suffered from fever more
Figure 1

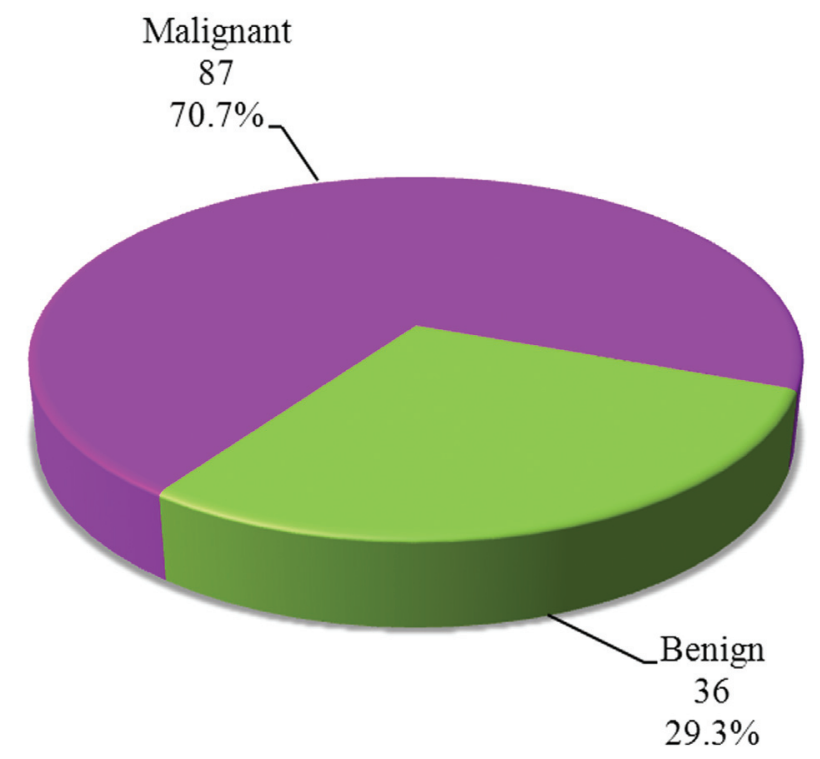

Distribution of the studied cases according to the final diagnosis.

significantly than patients with malignant pleural effusion. The CT findings were compared between benign and malignant groups (Table 3). The presence of pleural thickening, pleural nodules, pleural masses, lung parenchymal nodules, or masses, in addition to isolated mediastinal pleural thickening, were significantly more common in the malignant group, whereas the presence of parenchymal consolidation was in favor of benign disease. The comparison between the CT score sum in the benign versus the malignant group is presented in Fig. 2. The performance of the CT score in predicting the malignant nature of the pleural effusion was assessed in terms of sensitivity, specificity, PPV, NPV, and area under the ROC curve (Table 4 and Fig. 3). The CT score showed a sensitivity and a specificity of 70 and $66.7 \%$, respectively, with an NPV $83 \%$ and a PPV of $48 \%$, and the area under the ROC curve was 0.745 and the $95 \%$ confidence interval was between 0.650 and 0.839 $(P<0.001)$ using a cut-off value of at least 7 . Use of higher cut-off values resulted in reduced sensitivity and higher specificity.

\section{Discussion}

Of 123 (71\%) patients enrolled in this study, the majority of the patients were finally diagnosed with malignant effusion. We attribute this high prevalence of malignancy among our studied patients to the fact that the study was carried out in a tertiary care hospital; moreover, only patients who underwent medical thoracoscopy were enrolled, which might reflect indirect suspicion of malignancy from the clinician point of view in addition to the fact that $8 \%$ of our 
Table 2 Comparison between the two groups studied according to demographic and clinical data

\begin{tabular}{|c|c|c|c|c|c|}
\hline & Total $(n=123)[N(\%)]$ & Benign $(n=36)[N(\%)]$ & Malignant $(n=87)[N(\%)]$ & $\chi^{2}$ & $P$ \\
\hline \multicolumn{6}{|l|}{ Sex } \\
\hline Male & $59(48.0)$ & $22(61.1)$ & $37(42.5)$ & 3.523 & 0.061 \\
\hline Female & $64(52.0)$ & $14(38.9)$ & $50(57.5)$ & & \\
\hline \multicolumn{6}{|l|}{ Age (years) } \\
\hline Minimum-maximum & $20.0-86.0$ & $20.0-81.0$ & $26.0-86.0$ & $t=2.965^{\star}$ & $0.005^{*}$ \\
\hline Mean \pm SD & $54.25 \pm 13.43$ & $48.11 \pm 15.97$ & $56.79 \pm 11.39$ & & \\
\hline Median & 55.0 & 49.0 & 60.0 & & \\
\hline \multicolumn{6}{|l|}{ Asbestos exposure } \\
\hline No & $113(91.9)$ & $33(91.7)$ & $80(92.0)$ & 0.003 & ${ }^{\mathrm{FE}} P=1.000$ \\
\hline Yes & $10(8.1)$ & $3(8.3)$ & $7(8.0)$ & & \\
\hline \multicolumn{6}{|l|}{ Smoking history } \\
\hline No smoking & $90(73.2)$ & $27(75.0)$ & $63(72.4)$ & 0.087 & 0.768 \\
\hline Ex or current smoker & $33(26.8)$ & $9(25.0)$ & $24(27.6)$ & & \\
\hline \multicolumn{6}{|l|}{ History of previous cancer } \\
\hline No & $102(82.9)$ & $33(91.7)$ & $69(79.3)$ & 2.746 & 0.098 \\
\hline Breast cancer & $13(10.6)$ & $2(5.6)$ & $11(12.6)$ & 1.353 & ${ }^{\mathrm{FE}} P=0.342$ \\
\hline Bladder cancer & $2(1.6)$ & 0 & $2(2.3)$ & 0.841 & ${ }^{\mathrm{FE}} P=1.000$ \\
\hline Other types & $6(4.9)$ & $1(2.8)$ & $5(5.7)$ & 0.484 & ${ }^{\mathrm{FE}} P=0.670$ \\
\hline \multicolumn{6}{|l|}{ Complain } \\
\hline Cough & $55(44.7)$ & $11(30.6)$ & $44(50.6)$ & $4.128^{*}$ & $0.042^{*}$ \\
\hline Dyspnea & $119(96.7)$ & 35 (97.2) & $84(96.6)$ & 0.036 & ${ }^{\mathrm{FE}} P=1.000$ \\
\hline Pain & $68(55.3)$ & $11(30.6)$ & $57(65.5)$ & $12.590^{\star}$ & $<0.001^{*}$ \\
\hline Hemoptysis & $2(1.6)$ & 0 & $2(2.3)$ & 0.841 & ${ }^{\mathrm{FE}} P=1.000$ \\
\hline Fever & $12(9.8)$ & 7 (19.4) & $5(5.7)$ & $5.426^{\star}$ & ${ }^{\mathrm{FE}} P=0.039^{*}$ \\
\hline Weight loss & $60(48.8)$ & $11(30.6)$ & 49 (56.3) & $6.766^{*}$ & $0.009^{*}$ \\
\hline
\end{tabular}

${ }^{\star} P \leq 0.05$, statistically significant.

Table 3 Comparison between the two groups studied according to computed tomography findings

\begin{tabular}{|c|c|c|c|c|c|}
\hline Computed tomography findings & Total $(n=123)[N(\%)]$ & Benign $(n=36)[N(\%)]$ & Malignant $(n=87)[N(\%)]$ & $\chi^{2}$ & $P$ \\
\hline Mediastinal lymph nodes & $33(26.8)$ & 7 (19.4) & $26(29.9)$ & 1.414 & 0.234 \\
\hline Pleural Nodules & $32(26.0)$ & 0 & $32(36.8)$ & $17.898^{*}$ & $<0.001^{*}$ \\
\hline Pleural masses & $16(13.0)$ & $1(2.8)$ & $15(17.2)$ & $4.707^{\star}$ & ${ }^{\mathrm{FE}} P=0.037^{*}$ \\
\hline Isolated mediastinal pleural affection & $19(15.4)$ & 0 & $19(21.8)$ & $9.298^{\star}$ & $0.002^{*}$ \\
\hline Circumferential affection & $35(28.5)$ & $11(30.6)$ & $24(27.6)$ & 0.110 & 0.740 \\
\hline Pleural loculations & $21(17.1)$ & 8 (22.2) & $13(14.9)$ & 0.953 & 0.329 \\
\hline Pleural calcification & $1(0.8)$ & 0 & $1(1.1)$ & 0.417 & ${ }^{\mathrm{FE}} P=1.000$ \\
\hline Pericardial effusion & $4(3.3)$ & $1(2.8)$ & $3(3.4)$ & 0.036 & ${ }^{\mathrm{FE}} P=1.000$ \\
\hline \multicolumn{6}{|l|}{ Lung parenchyma } \\
\hline Mass & $15(12.2)$ & $1(2.8)$ & $14(16.1)$ & $4.215^{\star}$ & ${ }^{\mathrm{FE}} P=0.040^{*}$ \\
\hline Nodules & $26(21.1)$ & $1(2.8)$ & $25(28.7)$ & $10.292^{\star}$ & $0.001^{*}$ \\
\hline Consolidation & $21(17.1)$ & $12(33.3)$ & $9(10.3)$ & $9.504^{*}$ & $0.002^{*}$ \\
\hline \multicolumn{6}{|l|}{ Pleural thickening $>1 \mathrm{~cm}$} \\
\hline No & $41(33.3)$ & $15(41.7)$ & $26(29.9)$ & $19.667^{\star}$ & $<0.001^{*}$ \\
\hline Smooth & $40(32.5)$ & $19(52.8)$ & $21(24.1)$ & & \\
\hline Irregular & $42(34.1)$ & $2(5.6)$ & $40(46.0)$ & & \\
\hline
\end{tabular}

${ }^{\star} P \leq 0.05$, statistically significant.

studied patients reported heavy occupational exposure to asbestos. Other studies showed a lower prevalence of malignancy of about $25 \%[1,9]$.

We compared benign and malignant patient groups in terms of the individual parameters used in the CT scoring system and other CT findings (Table 2). Some parameters such as pleural thickening, pleural nodules, pleural masses, lung parenchymal nodules, and lung masses were seen significantly more frequently in the malignant group of patients in comparison with the benign group $(P<0.05)$. However, other parameters of the cardiac silhouette did not show any significant difference between both groups. The presence of isolated mediastinal pleural lesion, although not included in this score, was significantly more 


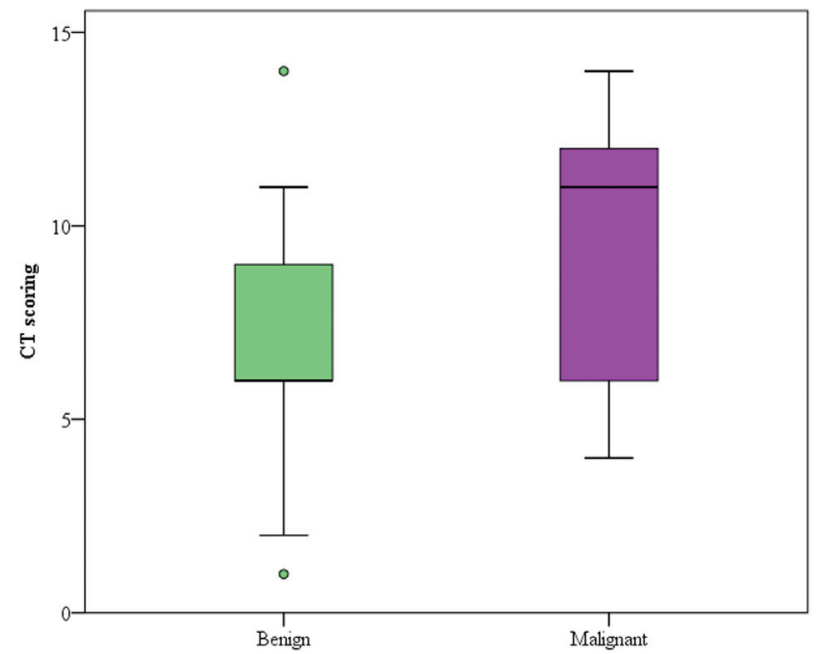

Comparison between the two groups studied according to computed tomography scoring.

\section{Figure 3}

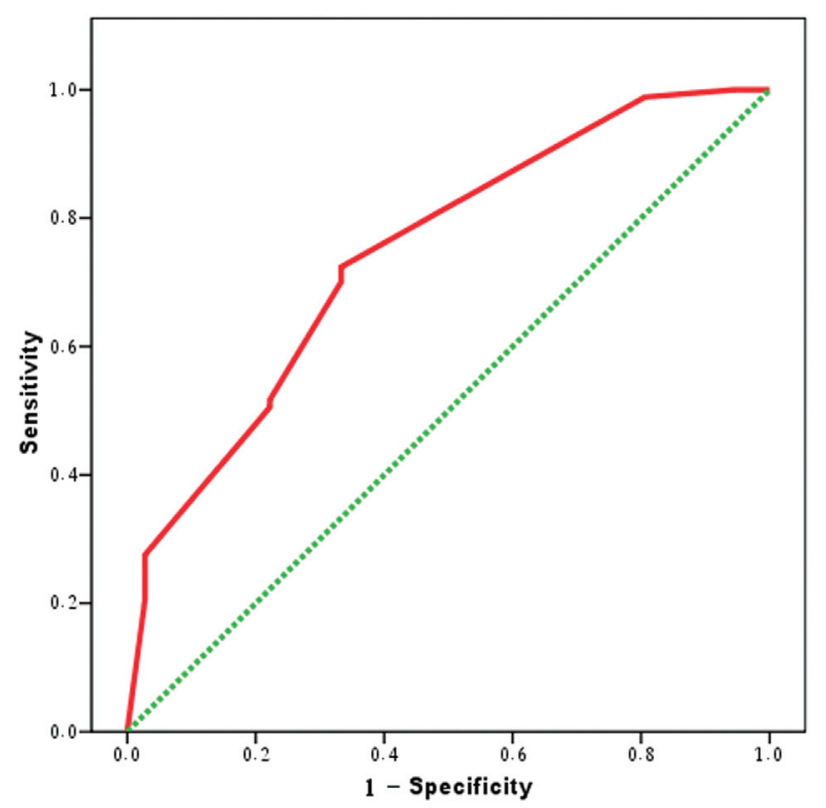

Receiver operating characteristic curve for computed tomography scoring as a predictor for malignancy.

frequent in patients with malignant effusion. The addition of this parameter might improve the performance of this CT scoring system. A number of previous studies found that CT findings of pleural nodularity, thickening of mediastinal pleura, and thickening of parietal pleural more than $1 \mathrm{~cm}$ and circumferential pleural thickening could predict the malignant cause of pleural effusion, with specificities ranging from 90 to $100 \%$ and sensitivities of $40-60 \%$ [10-12]. Another study investigated the sensitivity and specificity of CT in detecting the malignant cause of pleural effusion according to the radiologist impression before pathology results of thoracoscopic pleural
Table 4 Agreement (sensitivity, specificity, positive predictive value, and negative predictive value) for computed tomography scoring as a predictor for malignancy

\begin{tabular}{lcccc}
\hline Score cut-off & Sensitivity & Specificity & PPV & NPV \\
\hline$\geq 6$ & 72.41 & 66.67 & 84.0 & 50.0 \\
$\geq 7$ & 70.11 & 66.67 & 83.56 & 48.0 \\
$\geq 8$ & 70.11 & 66.67 & 83.56 & 48.0 \\
$\geq 9$ & 51.72 & 77.78 & 84.91 & 40.0 \\
$\geq 10$ & 50.57 & 77.78 & 84.62 & 39.44 \\
\hline
\end{tabular}

NPV, negative predictive value; PPV, positive predictive value. ${ }^{*} P \leq 0.05$, statistically significant.

biopsies, yielding a sensitivity of $68 \%$ and a specificity of $78 \%$ [7].

The score that we used in this study was derived and validated by Porcel et al. [8] Their study reported that this CT score using a sum score of 7 or more as a cut-off value yielded a sensitivity of $88 \%$, a specificity of $94 \%$ [95\% confidence interval (CI): 83-98\%], and an area under the receiver operating characteristics curve of 0.919 (95\% CI: 0.849-0.990). In contrast, in our study, using this cut-off value, this score showed lower performance as the sensitivity and specificity were 70 and $66.7 \%$, respectively, with an NPV of $83 \%$ and a PPV of $48 \%$, and the area under the ROC curve was 0.745. It is worth noting that unlike Porcel et al. [8], who enrolled only patients with unilateral effusion, in this study, we enrolled patients with both unilateral and bilateral effusion.

The results of this study showed that a simple score on the basis of CT findings is not very accurate or reliable. Use of special techniques might improve the performance of a CT-based score. A recent study reported that pleural attenuation is more evident for the delayed phase in comparison with the early phase of contrast-enhanced CT chest [13]. Another study validated a simple PET-CT score and showed that it can be useful to differentiate malignant from benign causes of pleural disease [14]. In this study, five PET$\mathrm{CT}$ parameters proved to be predictive of malignancy. These were unilateral lung nodules and/or masses with increased ${ }^{18} \mathrm{~F}$-FDG uptake; extrapulmonary malignancies; pleural thickening with increased 18Flourine-FluoroDeoxyGlucose $\left({ }^{18} \mathrm{~F}\right.$-FDG) uptake; multiple nodules or masses in one or both lungs with increased ${ }^{18} \mathrm{~F}$-FDG uptake; and increased pleural effusion ${ }^{18} \mathrm{~F}$-FDG uptake. No single individual CTPET parameter was predictive of malignancy, but was useful when combining more than one parameter together. A score of 4 or greater produced area under the curve, sensitivity, and specificity of 0.949 , 83.3, and $92.2 \%$, respectively, which is much higher than the AUC, sensitivity, and specificity in our study. 
However, a meta-analysis of 14 studies reported that PET imaging showed a significantly lower sensitivity for diagnosing malignant effusions than visual assessments ( 82 vs. $91 \% ; P=0.026$ ). Semiquantitative interpretations for identifying malignant effusions showed a sensitivity of $81 \%$, a specificity of $74 \%$, and area under the curve of 0.838 , concluding that the moderate accuracy of the technique using 18fluorodeoxyglucose is against its routine use to differentiate malignant from benign pleural effusions [15]. The same author developed and validated a CT scoring system for adults with parapneumonic pleural effusion that may allow clinicians to predict the need for chest tube drainage with good accuracy [16].

A large proportion of patients investigated for suspected malignant disease will show final results of malignancy irrespective of CT report impression; hence, the CT solely should not direct whether patients with pleural effusion should undergo further invasive pleural biopsies or not. This is in agreement with a retrospective series that suggests that approximately one-third of the patients with pleural malignancy may not show evident features of cancer on CT [17]. Moreover, in the study of Safwat et al. [18], although a sensitivity of $70 \%$ was found for CT chest to detect primary pleural tumors as 'high', CT chest failed to define parietal pleural invasion in $30 \%$, visceral pleural involvement in $60 \%$, and fibrinous septations in $10 \%$ of their enrolled patients.

Whether CT could save fragile patients, a more invasive procedure was an important consideration, if combined with close follow-up and observation. Adding mediastinal pleural lesion to CT parameters may improve the performance of this score in addition to considering other factors such as patient age, which was significantly higher in the malignant group of patients; in addition, important clinical data such as cough, chest pain, and significant weight loss were significantly more frequently reported also by the malignant group of patients, whereas the presence of fever was in favor of benign rather than malignant effusion.

In our opinion, creating a score that combines CT parameters with age and clinical data may be more informative and predictive of the final patient diagnosis than an isolated score based merely on CT. All the studies that discussed CT scores as predictors of malignancy showed low sensitivity even if the specificity was high. This is not accepted in a disease such as pleural effusion that is known that the majority of its patients will eventually turn out to be malignant. The strengths of our study were the fact that thoracoscopic pleural biopsy was considered the reference standard for the final diagnosis as well as the relatively large number of patients studied. The limitations of the current study are as follows: patients who were unfit or refused medical thoracoscopy were not included. Patients with extensive adhesions were also excluded as this was a contraindication for medical thoracoscopy. Morbidly obese patients whose weight exceeded that allowed to lay supine on $\mathrm{CT}$ table were also excluded $(>120 \mathrm{~kg})$. Furthermore, we only considered exudative pleural effusions, whereas malignancy can be associated with transudative effusion [19-21].

\section{Conclusion}

CT-based scoring system cannot predict the malignant nature of exudative effusion with high accuracy; neither can it help taking the decision to continue or stop further investigations.

\section{Financial support and sponsorship \\ Nil.}

\section{Conflicts of interest}

There are no conflicts of interest.

\section{References}

1 Porcel JM, Esquerda A, Vives M, Bielsa S. Etiology of pleural effusions: analysis of more than 3,000 consecutive thoracenteses. Arch Bronconeumol 2014; 50:161-165.

2 Gunnels J. Perplexing pleural effusions. Chest 1978; 74:390-393.

3 Rubins JB, Colice GL. Evaluating pleural effusions. Postgrad Med J 1999; 105:39-48.

4 Hooper C, Lee YC, Maskell N. Investigation of a unilateral pleural effusion in adults: British Thoracic Society pleural disease guideline2010. Thorax 2010; 65:ii4-ii17.

5 Clive AO, Kahan BC, Hooper CE, Bhatnagar R, Morley AJ, Zahan Evan N, et al. Predicting survival in malignant pleural effusion: development and validation of the LENT prognostic score. Thorax 2014; 69:1098-1104.

6 Traill ZC, Davies RJ, Gleeson FV. Thoracic computed tomography in patients with suspected malignant pleural effusions. Clin Radiol 2001; 56:193-196.

7 Hallifax RJ, Haris M, Corcoran JP, Leyakathalikhan S, Brown E, Srikantharaja D, et al. Role of CT in assessing pleural malignancy prior to thoracoscopy. Thorax 2015; 70:192-193.

8 Porcel JM, Pardina M, Bielsa S, González A, Light RW. Derivation and validation of a CT scan scoring system for discriminating malignant from benign pleural effusions. Chest 2015; 147:513-519.

9 Marel M, Zrustova M, Stasny B, Light RW. The incidence of pleural effusion in a well-defined region. Epidemiologic study in central Bohemia. Chest 1993; 104:1486-1489.

10 Leung AN, Müller NL, Miller RR. CT in differential diagnosis of diffuse pleural disease. Am J Roentgenol 1990; 154:487-492.

11 Scott EM, Marshall TJ, Flower CD, Stewart S. Diffuse pleural thickening: percutaneous CT-guided cutting needle biopsy. Radiology 1995; 194:867-870.

12 Davies HE, Nicholson JE, Rahman NM, Wilkinson EM, Davies RJ, Lee YC. Outcome of patients with nonspecific pleuritis/fibrosis on thoracoscopic pleural biopsies. Eur J Cardiothorac Surg 2010; 38:472-477. 
13 Arenas-Jiménez JJ, García-Garrigós E, Escudero-Fresneda C, SireraMatilla M, García-Pastor I, et al. Early and delayed phases of contrastenhanced CT for evaluating patients with malignant pleural effusion. Results of pairwise comparison by multiple observers. $\mathrm{Br} J$ Radiol 2018; 91:20180254.

14 Yang MF, Tong ZH, Wang Z, Yang MF, Tong ZH, Wang Z, et al. Development and validation of the PET-CT score for diagnosis of malignant pleural effusion. Eur J Nucl Med Mol Imaging 2019; 46:1457-1467.

15 Porcel JM, Hernandez P, Martinez-Alonso M, Bielsa S, Salud A. Accuracy of fluorodeoxyglucose-PET imaging for differentiating benign from malignant pleural effusions: a meta-analysis. Chest 2015; 147:502-512.

16 Porcel JM, Parinda M, Aleman C, Pallisa E, Light RW, Bielsa S. Computed tomography scoring system for discriminating between parapneumonic effusions eventually drained and those cured only with antibiotics. Respirology 2017; 22:1199-1204.
17 Tsim S, Stobo DB, Alexander L, Kelly C, Blyth KG. The diagnostic performance of routinely acquired and reported computed tomography imaging in patients presenting with suspected pleural malignancy. Lung Cancer 2017; 103:38-43.

18 Safwat T, Sharkawy S, Shoukri A, Mohamed S. Correlation between computed tomography of the chest and medical thoracoscopic findings in primary pleural tumors. Egypt $J$ Bronchol 2014; 8:32-37.

19 Walker S, Maskell N. Identification and management of pleural effusions of multiple aetiologies. Curr Opin Pulm Med 2017; 23:339-345.

20 Gonlugur TE, Gonlugur U. Transudates in malignancy: still a role for pleural fluid. Ann Acad Med Singapore 2008; 37:760-763.

21 Bintcliffe OJ, Hooper CE, Rider IJ, Finn RS, Morley AJ, Zahan E, et al. Unilateral pleural effusions with more than one apparent etiology. A prospective observational study. Ann Am Thorac Soc 2016; 13:1050-1056. 